\title{
Advances in Instrumental Colour Pass/Fail Analysis
}

\section{Westland $\mathbf{S}^{*}$ and Pan Q}

School of Design, University of Leeds, Leeds LS2 9JT, UK

\section{Background}

The requirement to be able to make a reliable decision about whether two samples are a visual colour match is an important commercial consideration for most textile production companies $[1,2]$. Traditionally visual pass/fail analysis was carried out by trained colourists. However, these visual decisions are subject to a number of problems which can make them unreliable. Approximately $8 \%$ of the male population have a colour vision deficiency (known colloquially as colour blindness) which means that pairs of samples may be a visual match to them despite appearing to be very different to other so-called normal observers [3]. Some variation in colour vision also is found in normal observers [4] and the effect of the viewing environment can affect visual decisions. Although the use of high-quality viewing cabinets can reduce variation in pass/fail decisions even the colour of the background in the cabinet against which the pair of samples are viewed can greatly affect the magnitude of the visual difference (the 'crispening' effect) [5]. In 1953 an analysis of 287 pairs of samples were visually assessed by 8 trained colourists [6] and a later analysis of these data showed that $24.5 \%$ of the pairs that should pass were rejected and $13.3 \%$ of the pairs that should be rejected were passed [1]. Several such studies have since been carried out and it is widely understood that as many as $25 \%$ 'wrong decisions' are made by professional colourists when making visual pass/ fail decisions [7]. This variability in the pass/fail decision is potentially costly and instrumental methods have been available for at least 50 years. This paper considers the current state of instrumental colourdifference evaluation and highlights some best practice.

\section{The CIE System of Colorimetry}

The CIE (Commission Internationale d'Eclairage) system of colorimetry was introduced in 1931 [8]. It provides a mathematical framework to allow measurements of spectral reflectance to be converted into CIE XYZ tristimulus values given a standard relative spectral power distribution for the illuminant. The principle of the CIE system is simple; if two samples have the same XYZ values then they will be a visual match to an average observer if the samples are viewed in standardised conditions under the illumination that matches the illuminant under which the XYZ values were calculated. However, in practice this perfect scenario is never met and in the middle of the $20^{\text {th }}$ Century a practical problem emerged, which was to decide on the pass/ fail tolerance (how close the XYZ values of two samples need to be for the two samples to be deemed a visual match). Many studies revealed that the CIE XYZ colour space (and its associated chromaticity diagram) is not perceptually uniform. In other words, the same magnitude of differences in $\mathrm{X}, \mathrm{Y}$ and $\mathrm{Z}$ result in greater or smaller visual differences depending upon the colours of the samples being considered (for example, very small changes in the blue region may be barely noticeable whereas much larger changes in the green region would not be visible). The problem of visual uniformity was one of the reasons why the CIELAB system was introduced in 1976 [9]. The CIELAB colour space is a non-linear transformation of the original XYZ system and is notably more visual uniform. Colour differences in CIELAB are expressed in terms of a colour difference, $\Delta \mathrm{E}$, which is calculated as the Euclidean distance in CIELAB space between the two points that represent two samples (eqn. (1)). In eqn. (1) the $\Delta \mathrm{E}$ is calculated from the differences in $L$, $a$ and $b$ between the two samples (where $L^{*}, a^{*}$ and $b^{*}$ are the CIELAB colour coordinates).

$$
\Delta \mathrm{E}=\left(\Delta \mathrm{L}^{* 2}+\Delta \mathrm{a}^{* 2}+\Delta \mathrm{a}^{* 2}+\Delta \mathrm{b}^{* 2}\right)^{0.5} \ldots
$$

Unfortunately, although CIELAB was a substantial improvement on the CIE XYZ system it is only approximately visually uniform and cannot be used for the Holy Grail of colour measurement - singlenumber pass/fail colour difference (where a fixed tolerance, such as $\Delta \mathrm{E}=1$, can be used as the pass/fail limit no matter what the colour of the pair of samples being considered or the nature of the colour difference between them) [9]. In fact, it has been stated that the performance of the CIELAB colour-difference formula for instrumental pass/fail would give results no better than what one would expect from a single observer [9].

\section{Colour Difference Equations}

The last 50 years has seen substantial developments in instrumental colour-difference evaluation and the introduction of many new equations. The JPC79 colour-difference equation was introduced [10] and was later modified to produce the CMC equation [11]. The CMC equation, published in 1984, weights differences in lightness, chroma and hue according to the colour of the standard sample of the pair based upon an equation that was fitted to the visual performance of a number of observers. Note that the CIELAB equation can be expressed in polar coordinates rather than the Cartesian coordinates of eqn. (1), thus:

$$
\Delta \mathrm{E}=\left(\Delta \mathrm{L}^{* 2}+\Delta \mathrm{C}^{* 2}+\Delta \mathrm{H}^{* 2}\right)^{0.5}
$$

Although CIELAB colour differences can be calculated from either Cartesian or polar coordinates, most of the optimized equations (such as JPC79 and CMC) that have been developed since are based on the polar representation of chroma and hue only. Eqn. (3) shows the generic form of an optimized colour-difference equation:

$$
\Delta \mathrm{E}_{\mathrm{O}}=\left(\left(\Delta \mathrm{L}^{*} / \mathrm{W}_{\mathrm{L}}\right)^{2}+\left(\Delta \mathrm{C}^{*} / \mathrm{W}_{\mathrm{C}}\right)^{2}+\left(\Delta \mathrm{H}^{*} / \mathrm{W}_{\mathrm{h}}\right)^{2}\right)^{0.5}
$$

where $\Delta \mathrm{E}_{0}$ is the optimized colour difference and $\mathrm{W}_{\mathrm{L}}, \mathrm{W}_{\mathrm{C}}$ and $\mathrm{W}_{\mathrm{h}}$ are weighting factors that are calculated in various ways depending upon the equation. The JPC79 and CMC colour difference formulae both take the generic form of eqn. (3) but calculate the weighting factors in different ways. A further advancement was made in 1987 with the development of the BFD colour-difference equation which was a refinement of the CMC equation [12]. Meanwhile, some researchers doubted whether the complexity of the BFD equation was justified and a much simpler equation, known, as CIE94 was introduced [13]. The

*Corresponding author: Westland S, School of Design, University of Leeds, Leeds LS2 9JT, UK, Tel: +44 113243 1751; E-mail: S.Westland@leeds.ac.uk

Received September 01, 2017; Accepted September 11, 2017; Published September 30, 2017

Citation: Westland S, Pan Q (2017) Advances in Instrumental Colour Pass/Fail Analysis. J Textile Sci Eng 7: 321. doi: 10.4172/2165-8064.1000321

Copyright: (c) 2017 Westland S, et al. This is an open-access article distributed under the terms of the Creative Commons Attribution License, which permits unrestricted use, distribution, and reproduction in any medium, provided the original author and source are credited. 
CIEDE2000 colour-difference was published in 2001 by the CIE based on extensive research $[14,15]$. In addition to the lightness, chroma and hue weighting functions, CIEDE2000 includes an interactive term between chroma and hue differences for improving the performance in the blue region of colour space and a CIELAB $\mathrm{a}^{*}$ term for improving the performance for grey colours [14].

A number of studies have compared the relative performance of the optimized colour-difference equations [16-19] and the outcome can sometimes depend upon the dataset that is used for the evaluation and the metric that is used to assess performance. However, for small colour differences (which are defined as those where the CIELAB $\Delta \mathrm{E}<5)$ there is overwhelming evidence that modern formula such as CIEDE2000 outperform CIELAB. The CIEDE2000 formula is widely regarded as the most accurate colour-difference formula for smallcolour differences and in one study, for example, outperformed both CMC and CIE94 by a large margin [14].

\section{Best Practice and Outlook}

The current CIE recommendation for the instrumental evaluation of small colour differences is the CIEDE2000 formula and is officially adopted as the CIE colour-difference equation [14]. For large colour differences the recommendation is still CIELAB; in part, because of the research that has been carried out to develop colour-difference equations has been with small colour differences since these are the differences that are most commercially significant. Rather less research has been carried out with supra-threshold colour differences. Despite the fact that the CIELAB colour-difference equation could be regarded as technology that is more than 40 years odd, in the author's experience it is still widely used in industry and many quality control departments are unaware of CIEDE2000 or unfamiliar with it. One factor that may have inhibited the uptake of the optimized colour-difference equations (from JPC79 to CIEDE2000) is that they are based on the polar coordinates of CIELAB colour space rather than the Cartesian coordinates. Cartesian geometry is so ubiquitous at school-level education that it is barely necessary to refer to it by name. However, an engagement with the polar coordinates of chroma and hue should be encouraged. There is a reason why the optimized equations since 1976 have been based on chroma and hue rather than $\mathrm{a}^{*}$ and $\mathrm{b}^{*}$ and that is that it is much easier to relate changes in the polar coordinates to visual changes. Conversely, the coordinates $\mathrm{a}^{*}$ and $\mathrm{b}^{*}$ on their own each confound the variables of chroma and hue [20].

Although the CIEDE2000 should be preferred to CIELAB as a colour-difference equation for use in most industrial applications this is no way suggests that CIELAB as a colour space is soon to be replaced. CIELAB has been a standard colour space for more than 40 years and remains so. Indeed, all of the optimized colour-difference equations that have been discussed in this paper are based on CIELAB. Recent research, however, is exploring other spaces (which are more uniform than CIELAB) in which to base colour-difference metrics [20]. Unlike, CIE XYZ, CIELAB is a colour-appearance space; however, it is not a particular good one. Advances in colour-appearance research have led to a number of new models such as CIECAM02 which can describe many colour-appearance phenomena that are beyond CIELAB. It is likely that in the future we will see more colour-difference equations based on CIECAM02 and related colour spaces and that CIEDE2000 will not be the final word in instrumental evaluation of colour difference.

\section{References}

1. McLaren K (1970) Colour Passing-Visual or Instrumental? Coloration
Technology 86: 389-392.

2. McDonald R (1980) Industrial Pass/Fail Colour Matching Part 1-Preparation of Visual Colour-matching Data. Coloration Technology 96: 372-376.

3. Birch J (2012) Worldwide prevalence of red-green color deficiency. Birch J. J Opt Soc Am A Opt Image Sci Vis 29: 313-320.

4. Deeb SS (2005) The molecular basis of variation in human color vision. Clinical Genetics 67: 369-377.

5. Whittle $P$ (1992) Brightness, discriminability and the "crispening effect". Vision Research 32: 1493-1507.

6. Davidson HR, Friede E (1953) The size of acceptable color differences. Journal of the Optical Society of America 43: 581-589.

7. Robinson FD (1969) Acceptability of colour matches-hull section co-Operative research project. Journal of the Oil \& Colour Chemists Association 52: 15.

8. Westland S, Ripamonti C, Cheung V (2012) Computational colour science using MATLAB ( $2^{\text {nd }}$ edn. $)$, Wiley.

9. Rigg B (1995) Colour-difference formulae-recent developments. Coloration Technology 111: 267-271.

10. McDonald R (2008) Industrial pass/fail colour matching. Part III-Development of a Pass/Fail Formula for use with Instrumental Measurement of Colour Difference. Coloration Technology 96: 486-497.

11. Clarke FJ, McDonald R, Rigg B (2008) Modification to the JPC79 colourdifference formula. Coloration Technology 100: 128-132.

12. Luo MR, Rigg B (1987) BFD (l: c) colour-difference formula Part 1- Development of the formula. Coloration Technology 103: 86-94.

13. McDonald R, Smith KJ (1995) CIE94 - a new colour-difference formula Coloration Technology 111: 376-379.

14. Luo MR, Cui G, Rigg B (2001) The development of the CIE 2000 colourdifference formula: CIEDE2000. Color research and application 26: 340-350.

15. CIE Publication (2004) Colorimetry ( $3^{\text {rd }}$ edn.), CIE Central Bureau.

16. Oglesby S (1995) The effectiveness of CIE94 compared with the CMC equation Coloration Technology 111: 380-381.

17. Heggie D, Wardman RH, Luo MR (1996) A comparison of the colour differences computed using the CIE94, CMC (I: c) and BFD (I: c) formulae. Journal of the Society of Dyers and Colourists 112: 264-269.

18. Melgosa M (2000) Testing CIELAB-based color-difference formulas. Color Research \& Application 25: 49-55.

19. Wang H, Cui G, Luo MR, Xu H (2012) Evaluation of colour-difference formulae for different colour-difference magnitudes. Color Research \& Application 37: 316-325.

20. Luo MR, Cui G, Li C (2006) Uniform colour spaces based on CIECAM02 colour appearance model. Color research and application 31: 320-330. 